\title{
Line driven winds, ionizing fluxes and UV-spectra of hot stars at extremely low metallicity
}

\author{
Rolf-Peter Kudritzki \\ Institute for Astronomy, University of Hawaii at Manoa, \\ 2680 Woodlawn Drive, Honolulu, HI 96822, USA
}

\begin{abstract}
Wind models of very massive stars with metallicities in a range from $10^{-4}-1.0 Z_{\odot}$ are presented using a new treatment of radiation driven winds with depth dependent radiative force multipliers and a comprehensive list of more than two million of spectral lines in non-LTE. The models yield mass-loss rates, wind velocities, wind momenta and wind energies as a function of metallicity and can be used to discuss the influence of stellar winds on the evolution of very massive stars in the early universe and on the interstellar medium in the early phases of galaxy formation. It is shown that the normal scaling laws, which predict stellar mass-loss rates and wind momenta to decrease as a power law with metal abundance break down at a certain threshold. The new wind models are applied to calculate ionizing fluxes and observable UV-spectra of very massive stars as a function of metallicity using the WM-basic code developed by Pauldrach et al. (2001), and the efffects of metallicity are discussed.
\end{abstract}

\section{Introduction}

There is growing evidence from numerical simulations that the evolution of galaxies in the early universe was heavily influenced by the formation of first generations of very massive stars (Abel et al. 2000, 2002; Bromm et al. 1999, 2001ab; Nakamura \& Umemura 2001). Such an early population of very massive stars at very low metallicities could contribute significantly to the ionization history of the intergalactic medium (Carr et al. 1984; Couchman \& Rees 1986; Haiman \& Loeb 1997; Bromm et al. 2001b). Very massive stars are also very likely the progenitors of Gamma-Ray bursts, which then - as tracers of the cosmic star formation history - might originate to a large fraction at very high reshift (Bromm \& Loeb 2002; Ciardi \& Loeb 2000; Lamb \& Reichart 2000). In addition, the extreme $\operatorname{Ly} \alpha$ emitting galaxies at high red redshift can be explained by an ionizing population of very massive stars at very low metallicity (Kudritzki et al. 2000; Rhoads \& Malhotra 2002; Malhotra \& Rhoads 2002).

In order to be able to make more quantitative predictions about the influence of such an extremely metal-poor population of very massive stars on their galactic and intergalactic environment, one needs to determine their physical properties during their evolution. A key issue in this regard is the knowledge about their radiation driven stellar winds. These winds have substantial effects on the structure of the radiating atmospheres. They dominate the density stratification and the radiative transfer through the presence of their macroscopic transonic velocity fields (see Kudritzki 1998, for a detailed review) and they 
modify the amount of the emergent ionizing radiation significantly (Gabler et al. 1989; 1991, 1992; Najarro et al. 1996). Winds have an extremely important influence on the evolution of massive stars by reducing the stellar mass continuously and by affecting evolutionary time-scales, chemical profiles, surface abundances and luminosities. Providing a significant input of mechanical and radiative momentum and energy into the ISM (or the environment of a GRB) together with the injection of nuclear processed material they can also play a crucial role for the evolution of galaxies. Last but not least, stellar winds provide beautiful spectroscopic tools to investigate the physical properties of galaxies through the analysis of broad stellar wind spectral line features easily detectable in the integrated spectra of starforming galaxies in the nearby and high-redshift universe (Pettini et al. 2000; Leitherer et al. 2001).

While the investigation of winds from massive stars in the solar neighborhood and the Magellanic Clouds has been the subject of extensive work (see Kudritzki \& Puls 2000, for a recent review), little is known so far about winds at very low metallicity. Since these winds are initiated and maintained through absorption of photospheric photon momentum by UV metal lines, we expect their strengths to decrease with decreasing metallicity. Theoretical work by $\mathrm{Ab}-$ bott (1982), Kudritzki et al. (1987), Leitherer et al. (1992) and, most recently, by Vink et al. (2001) for normal OB-stars in a metallicity range from 3.0 solar to $1 / 30$ solar predicts that mass-loss rates and terminal velocities scale with $\left(Z / \mathrm{Z}_{\odot}\right)^{n}$, where $n=0.5-0.8$ for $\dot{M}$ and 0.15 for $v_{\infty}$. Observational spectroscopic studies of massive stars in the Magellanic Clouds confirm that this theoretical prediction is basically correct for metallicities ranging to $0.1 \mathrm{Z}_{\odot}$ (see Kudritzki \& Puls 2000, but also Vink et al. 2001).

The objective of the review presented here is to discuss the mechanism of radiative line driving and the corresponding properties of the winds of possible generations of very massive stars (as predicted by the numerical simulations mentioned above) at extremely low metallicities and to investigate the principal influence of these winds on ionizing fluxes and observable ultraviolet spectra. The very low metallicities require the development of a new approach to calculate the wind dynamics, which introduces depth dependent force multipliers to represent the radiative line acceleration, a new formulation of the critical point equations is developed and a new iterative solution algorithm for the complete stellar wind problem. This new approach allows to calculate wind models within a few seconds on a workstation for every hot star with specified effective temperature, mass, radius and abundances.

Our review concentrates on mass-loss through radiation driven winds only. As is well known, very massive stars are pulsationally unstable, which might contribute to stellar-mass loss, in particular at low metallicity when the contribution of the radiative driving to the winds decreases. However, very recently, Baraffe et al. (2001) have studied this problem and found that the possible effects of pulsation on mass-loss are much weaker for very massive stars with low metallicity than for those with solar metallicity. It is thus very likely that the mechanism of radiative line driving remains still important in the metallicity range discussed here, although pulsational instability will probably lead to an additional mass-loss contribution for our lowest metallicity models. 


\section{Radiative line acceleration at very low metallicities}

The crucial term in the hydrodynamics of radiation driven winds is the radiative line acceleration, which can be expressed in units of the Thomson acceleration $g_{\text {rad }}^{\text {Th }}$

$$
g_{\mathrm{rad}}^{\text {lines }}=g_{\mathrm{rad}}^{\mathrm{Th}} C F\left(r, v, \frac{d v}{d r}\right) M(t) .
$$

$C F\left(r, v, \frac{d v}{d r}\right)$ is the finite cone angle correction factor, which takes into account that a volume element in the stellar wind is irradiated by a stellar disk of finite angular diameter rather than a point source. $M(\mathrm{t})$ is the line force multiplier which gives the line acceleration in units of Thomson scattering. In the Sobolev approximation the contribution of all spectral lines $i$ at frequencies $\nu_{i}$ and at spectral luminosities $L_{\nu_{i}}$ to the line force multiplier is given by

$$
M(t)=\frac{v_{\text {therm }}}{c} \frac{1}{t} \sum_{i} \frac{\nu_{i} L_{\nu_{i}}}{L}\left(1-e^{-\tau_{i}}\right)
$$

where $v_{\text {therm }}$ is the thermal velocity of hydrogen, $c$ the speed of light, $\tau_{i}=$ $k_{i} t(r)$ the local (Sobolev) optical depth with $k_{i} \propto \frac{h_{l}}{n_{e}} f_{l u} \lambda_{i}$ the line strength and $t(r)=n_{e} \sigma_{e} \frac{v_{\text {therm }}}{d v / d r}$ the optical depth parameter. $M(t)$ is usually fitted by the parametrization $(W(r)$ is the geometrical dilution factor of the radiation field)

$$
M(t)=\hat{k} t^{-\alpha} \hat{n}^{\delta}, \quad \hat{n}=\frac{n_{e}(r)}{W(r)} / 10^{11} \mathrm{~cm}^{-3} .
$$

$\hat{k}, \alpha, \delta$ are the force multiplier parameters (fmps). This parametrization has the advantage that it allows very fast numerical solutions and very precise analytical approximations of the complex hydrodynamical problem of line driven winds (for details, see Castor et al. 1975; Abbott 1982; Pauldrach et al. 1986; Kudritzki et al. 1988; and Kudritzki 1998 and references therein). However, it does only work as long as the fmps are really constant in the atmosphere. As shown by Kudritzki et al. (1998) and Kudritzki (2002), Eq. 2 fails badly to reproduce the line force multiplier $M$ in the full parameter plane of $t$ and $\hat{n}$ for early type stars and becomes particularily bad at very low metallicities. A much more accurate representation is given by the form

$$
\log M(t)=\log \hat{k}-\alpha_{o}\left(1+\alpha_{1} \log t\right) \log t+\delta_{o}\left(1+\delta_{1} \log \hat{n}\right) \log \hat{n}+\gamma \log t \log \hat{n}
$$

which allows for the fact that the fmps depend on optical depth and density themselves. This improved parametrization requires a new treatment of the solution of the stellar wind equation of motion including the transition through the singularity of the critical point and the constraint that the photospheric radius has to be at contiuum optical depth equal to $2 / 3$ (see Kudritzki 2002, for a detailed description).

\section{Wind models for very massive stars at low metallicity}

Using the new treatment of line driven winds, Kudritzki (2002) has carried out the first calculations of stellar winds for very massive hot stars at very low 

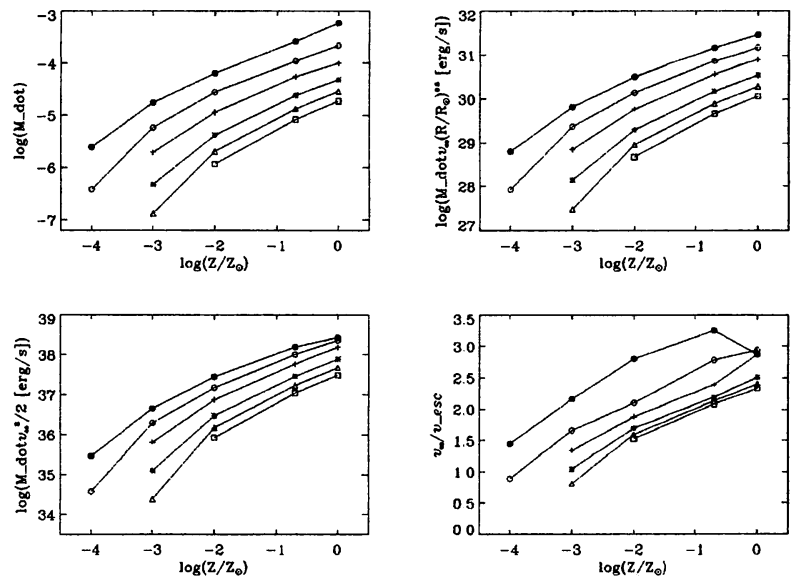

Figure 1. Stellar wind properties as a function of metallicity for models of different luminosity with $\log L / \mathrm{L}_{\odot}=7.03$ (solid circles), 6.91 (open circles), 6.76 (plus signs), 6.57 (asterisks), 6.42 (triangles), 6.30 (squares). Upper left: mass-loss rates; upper right: modified stellar wind momentum; lower left: wind energy; lower right: terminal velocity in units of photospheric escape velocity.

metallicity in the mass range between 100 to $300 \mathrm{M}_{\odot}$, which according to the numerical simulation is the preferred mass range of star at very low metallicity. He concentrated on an effective temperature range comparable to the hottest and most massive observed O-type stars, i.e., $60000 \mathrm{~K}$ to $40000 \mathrm{~K}$, which is only a mild extrapolation away from a stellar parameter regime, where the theory has been tested at Galactic and SMC metallicity. The calculations can, however, easily be extended to higher effective temperatures to cover the shift of zero age main sequences in the H-R diagram with decreasing metallicity.

Figure 1 displays stellar mass-loss rates, wind momenta and energies and terminal velocities as a function of metallicity. Very obviously, at metallicities beyond $Z / \mathrm{Z}_{\odot}=0.01$ all stellar wind quantities decrease stronger than simple power laws. This has important repercussions for the role of winds in stellar evolution, but also for the energy input into the ISM in early evolutionary stages of galaxies and the envelop structures around exploding hypernovae and their shocks leading to GRB afterglows at high redshifts.

\section{Ionizing fluxes and stellar spectra}

With the stellar wind structures and parameters specified Kudritzki (2002) calculated detailed atmospheric models together with stellar energy distributions and synthetic spectra using the approach of Unified Model Atmospheres as developed at Munich University Observatory over the last 15 years. These model atmospheres are in non-LTE, radiative equilibrium, spherically extended, include the effects of stellar winds and account for more than 4 millions of metal lines in non-LTE originating from more than 150 metal ions. Detailed atomic models with accurate atomic data are set up for every ion, for which the equations of 

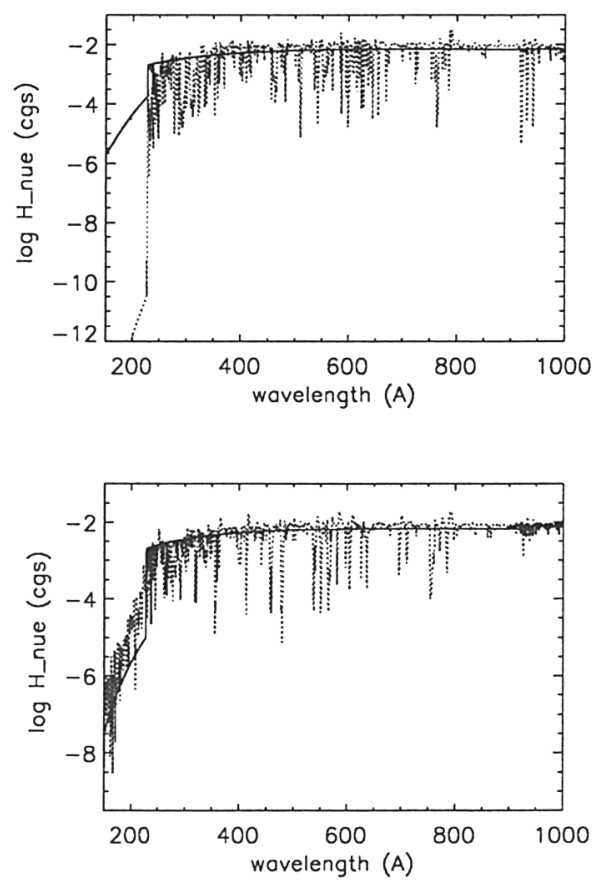

Figure 2. Logarithm of emergent EUV/FUV Eddington flux $\mathrm{H}_{\nu}$ as a function of wavelength for models with $T_{\text {eff }}=60000 \mathrm{~K}$ and two metallicities, $Z / \mathrm{Z}_{\odot}=1.0$ (dotted) and $10^{-4}$ (solid). Upper panel: $\log L / \mathrm{L}_{\odot}=6.91$; lower panel: $\log L / \mathrm{L}_{\odot}=6.57$. Note the dramatic differences in the He II continuum shortward of $227 \AA$. See Kudritzki (2002) for further discussion.

statistical equilibrium are solved consistently and simultaneously with the radiative transfer in each line and ionization transition in a highly iterative algorithm. Multi-line absorption is included in the radiative transfer and in the radiative equilibrium, which means that the effects of line-blanketing and -blocking are fully taken into account. After convergence spectra and energy distributions are calculated including all the spectral lines. The code is thus ideally suited to demonstrate the transition from solar to very low metallicity. In addition, despite the complexity of the atomic models and the radiative transfer algorithms the code is extremely fast and produces a converged model on a laptop or PC in a few hours. It is public available and can be downloaded from the Munich University Observatory website ${ }^{1}$. For details we refer the reader to the original publication by Pauldrach et al. (2001).

Figure 2 demonstrates the effects of metallicity on the EUV and FUV spectral energy distribution. Longward of $228 \AA$, in the H, He I, O II, Ne II, C III ionizing continuum, the influence of metal line opacity is very similar at both luminosities. Increased metallicity decreases the emergent flux within the metal lines because of enhanced line blocking, but increases the flux emitted in the

\footnotetext{
${ }^{1}$ http://ww . usm. uni-muenchen.de/people/adi/adi.html
} 

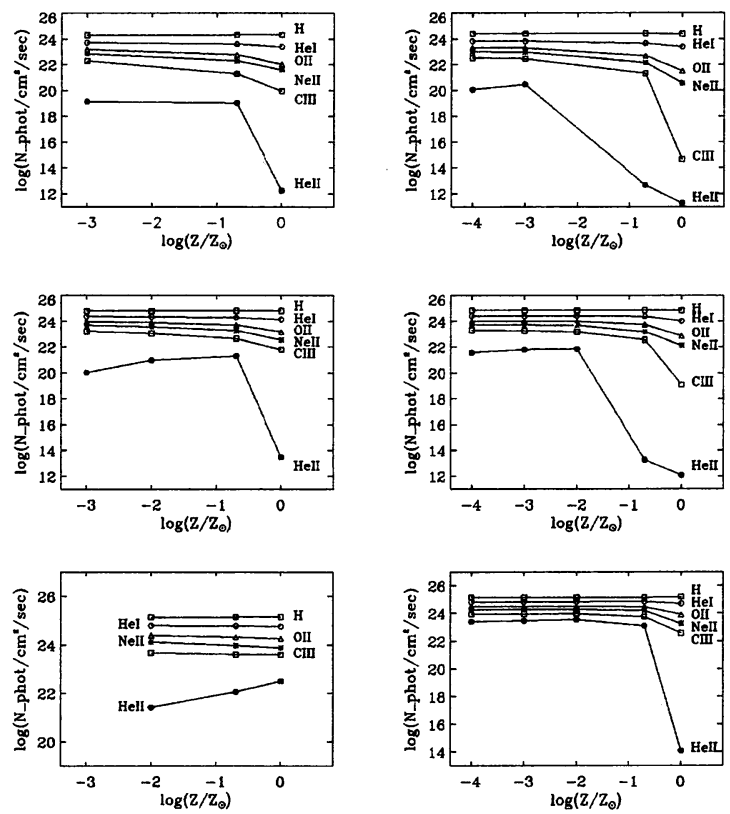

Figure 3. Logarithm of the emitted number of ionizing photons as a function of stellar metallicity (see text). Left: $\log L / \mathrm{L}_{\odot}=6.42 ;$ right: $\log L / \mathrm{L}_{\odot}=7.03$. Top panels: $T_{\mathrm{eff}}=40000 \mathrm{~K}$; middle panels: $T_{\mathrm{eff}}=50000 \mathrm{~K}$; bottom panels: $T_{\text {eff }}=60000 \mathrm{~K}$ (from Kudritzki 2002).

continuum windows with reduced line opacity because of the back-warming effects of line-blanketing. The balance between the different influence of blocking and blanketing will, therefore, determine how the ionizing properties of these stars are affected by metallicity (see below).

Shortward of $228 \AA$, in the He II ionizing continuum, metallicity has a dramatic influence on the size of the He II absorption edge and, thus, on the ionizing flux. However, this influence is less related to the effects of line-blanketing and -blocking rather than to the strengths of the stellar winds correlated with metallicity (see Gabler et al. 1989, 1991, 1992 for a detailed explanation).

Figure 3 summarizes the effects of metallicity on the ionizing properties of very massive stars. To characterize the wavelength dependence of the ionizing radiation we display the number of photons being able to ionize $\mathrm{H}$ (ionization edge at $911 \AA), \mathrm{He}$ I (504 $\AA$ ), O II (353 $\AA$ ), Ne III (303 $\AA$ ), C III (259 $)$ and He II (228 $\AA$ ). The dependence on metallicity of these photon numbers varies significantly with the ionization edge approaching the limit of $\mathrm{He}$ II ionization. While the number of $\mathrm{H}$ photons remains almost constant and the effects of metal lineblocking and -blanketing balance out over the relatively wide spectral range from the hydrogen to the He II absorption edge, the number of C III photons decreases strongly with increasing metallicity, because line blocking dominates the remaining wavelength interval towards the He II absorption edge. The He II ionizing photons reflect a more complex behavior as discussed above and vary dramatically as a function of metallicity and luminosity. 

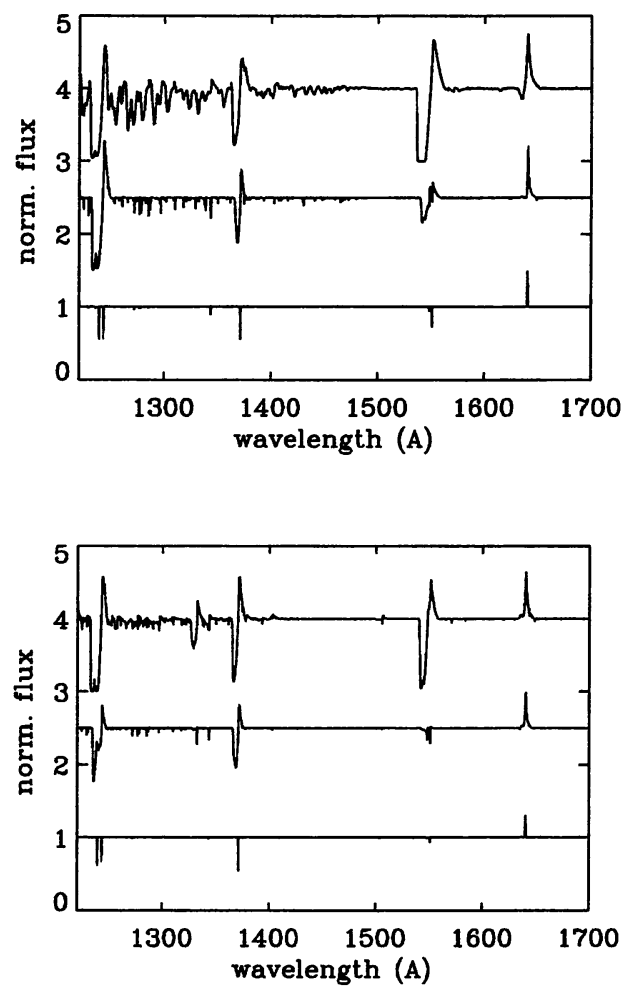

Figure 4. Continuum rectified UV-spectra longward of Ly $\alpha$ for models with $\log L / \mathrm{L}_{\odot}=6.91$ and $T_{\text {eff }}=50000 \mathrm{~K}$ (top panel) and $60000 \mathrm{~K}$ (bottom panel). Each panel displays three metallicities, $Z / \mathrm{Z}_{\odot}=0.2($ top $), 10^{-2}$ (middle), and $10^{-4}$ (bottom). The strongest line features, which remain present at the lowest metallicity, are N v $\lambda 1250 \AA, \mathrm{O} v \lambda 1371 \AA, \mathrm{C}$ IV $\lambda 1550 \AA$ and He II $\lambda 1640 \AA$ (see Kudritzki 2002, for details).

It is also interesting to calculate synthetic FUV and UV spectra as a function of metallicity. As we know well from IUE, HST, ORFEUS and FUSE observations of massive stars in the Galaxy and the Large and Small Magellanic Cloud, the observed spectra in this spectral range are heavily blended by a dense forest of slightly wind affected pseudo-photospheric metal absorption lines superimposed by broad P-Cygni and emission line profiles of strong lines formed in the entire wind. For stars at very low metallicity, the interesting question to investigate is to find the metallicity range, where the UV stellar wind features and the photospheric metal lines absorption lines start to disappear. Figure 4 gives an overview for $\log L / \mathrm{L}_{\odot}=6.91$, where the winds are relatively strong. The stellar wind lines (Nv $\lambda 1240 \AA$, OV $\lambda 1371 \AA, \mathrm{C}_{\mathrm{IV}} \lambda 1550 \AA$, He II $\lambda 1640 \AA$ ) remain clearly visible down to $Z / \mathrm{Z}_{\odot}=10^{-2}$, where the absorption forest has already started to disappear. For lower metallicity, the stellar wind character of these lines disappears, but they are still detectable as photospheric absorption or emission lines. This means that for starbursting galaxies at very high redshift and possibly very low metallicities, as eventually observable with 
NGST in the IR, there is still diagnostic information available to estimate chemical abundances and further properties such as the Initial Mass Function and the star formation rate of the integrated stellar population.

\section{References}

Abbott, D.C. 1982, ApJ 259, 282

Abel, T., Bryan, G.L., Norman, M.L. 2000, ApJ 540, 39

Abel, T., Bryan, G.L., Norman, M.L. 2002, Science 295, 93

Baraffe, I., Heger, A., Woosley, S.E. 2001, ApJ 550, 890

Bromm, V., Coppi, P.S., Larson, R.B. 1999, ApJ (Letters) 527, L5

Bromm, V., Ferrara, A., Coppi, P.S., Larson, R.B. 2001a, MNRAS 328, 969

Bromm, V., Kudritzki, R.-P., Loeb, A. 2001b, ApJ 552, 464

Bromm, V., Loeb, A. 2002, ApJ 575, 111

Carr, B.J., Bond, J.R., Arnett, W.D. 1984, ApJ 277, 445

Castor, J.I., Abbott, D.C., Klein, R.I. 1975, ApJ 195, 157

Ciardi, B., Loeb, A. 2000, ApJ 540, 687

Couchman, H.M.P., Rees, M.J. 1986, MNRAS 221, 53

Gabler, R., Gabler, A., Kudritzki, R.-P., et al. 1989 A\&A 226, 162

Gabler, R., Kudritzki, R.-P., Méndez, R.H. 1991, A\&A 245, 587

Gabler, R., Gabler, A., Kudritzki, R.-P., Méndez, R.H. 1992, A\&A 265, 656

Haiman, Z., Loeb, A. 1997, ApJ 483, 21

Kudritzki, R.-P., Yorke, H.W., Frische, H. 1988, in: Y. Chmielewski \& T. Lanz (eds.), Radiation in Moving Gaseous Media, Proc. 18th Advanced Course of the Swiss Society of Astrophysics and Astronomy (Saas-Fee Courses), pp. 1-192

Kudritzki, R.-P. 1998, in: A. Aparicio, A. Herrero \& F. Sánchez (eds.), Stellar Astrophysics for the Local Group, Proc. VIII Canary Islands Winter School on Stellar Astrophysics (Cambridge: CUP), p. 149

Kudritzki, R.-P., Pauldrach, A.W.A., Puls, J. 1987, A\&A 173, 293

Kudritzki, R.-P., Springmann, U., Puls, J., et al. 1998, in: I.D. Howarth (ed.), BoulderMunich II: Properties of Hot Luminous Stars, ASP-CS 131, 299

Kudritzki, R.-P., Puls, J. 2000, Ann. Review Astron. Astrophys. 38, 613

Kudritzki, R.-P. 2002, ApJ 577, 389

Lamb, D.Q., Reichart, D.E. 2000, ApJ 536, 1

Leitherer, C., Robert, C., Drissen, L. 1992, ApJ 401, 596

Leitherer, C., Leão, J.R.S., Heckman, T.M., et al. 2001, ApJ 550, 724

Malhotra, S., Rhoads, J.E. 2002, ApJ (Letters) 565, L71

Najarro, F., Kudritzki, R.-P., Cassinelli, J.P., et al. 1996, A\&A 306, 892

Nakamura, F., Umemura, M. 2001, ApJ 548, 19

Pauldrach, A.W.A., Puls, J., Kudritzki, R.-P. 1986, A\&A 164, 86

Pauldrach, A.W.A., Lennon M., Hoffmann, T.L., Sellmaier, F., Kudritzki, R.-P., Puls, J. 1998, in: I.D. Howarth (ed.), Boulder-Munich II: Properties of Hot Luminous Stars, ASP-CS 131, 258

Pauldrach, A.W.A., Lennon, M., Hoffmann, T.L. 2001, A\&A 375, 161; Erratum 2002, A\&A 395, 611

Pettini, M., Steidel, C.C., Adelberger, K.L., et al. 2000, ApJ 528, 96

Rhoads, J.E., Malhotra, S. 2002, ApJ (Letters) in press

Vink, J., de Koter, A., Lamers, H. 2001, A\&A 369, 574 


\section{Discussion}

OwockI: In the original CAK theory, the number of lines with optical depth unity or greater is usually very large, and so it is reasonable to fit the discrete distribution of lines as a continuous power law. In stability models, however, one finds that this leads to unbounded growth of velocity in rarefied gas, and so it is necessary to introduce a cutoff, corresponding to the strength of the strongest line. It seems to me that your turnover to low $\alpha$ just reflects that you are simply running out of thick lines, meaning that small number statistics take hold. That is, $\alpha \rightarrow 0$ simply means that all lines are optically thin.

KUDRITZKI: My new algorithm fits the depth dependent force multiplier parameters $\alpha(t)$ and $\delta(u, w, t)$ to the numerically calculated line force calculated as a sum over all lines. Thus $\alpha$ and $\delta$ refer to the line force and not to the line strength distribution function. But it is true that $\alpha \rightarrow 0$, as shown in some of my diagrams, means that at very low metallicity we are running out of optical thick lines. We are approaching the saturation limit of the line force in the optical thin case.

EL EID: Considering the very massive stars you are considering, why should the mass loss be only due to stellar wind? Other mechanisms could be also important.

KUDRITZKI: I completely agree. Pulsational instability may be playing a role. One has to compare all mechanisms which may contribute to mass loss. I wanted to estimate the contribution of stellar wind to the mass loss from these stars.

HEger: ( $i$ ) Can $Z$-dependence of the mass loss role be extended to $Z=0$ ? (ii) What is your opinion on continuum-driven wind (at $Z=0$ )?

KUDRITZKI: $(i)$ It can be extended to some minimum metallicity $Z_{\min }$ according to my formula. Below this value things become very difficult with line driving through He II lines only. I have, so far, not succeeded to calculate winds for this case. (ii) I have not encountered cases with continuum opacity plus Thomson scattering $g_{\mathrm{rad}}$ exceeding $g_{\mathrm{r}}$ so that the effective gravity is negative in the photosphere. But the instability recently found by Shaviv in pure Thomson scattering close to the Eddington-limit might lead to winds.

FELDMEIER: Do you feel that Abbott's description of ionization effects in line-driven winds via a factor electron density over dilution factor to the power of $\delta$ is still valid with $\delta$ being not very small? Maybe Eddington factors or wind temperatures should then also be included?

KUDRITZKI: In the range of my calculations this approach works very well and represents the line force very accurately. I also refer to the paper by Puls et al. 2000, who discuss very carefully why you can encounter cases of very high $\delta$.

LEITHERER: Can you express the metallicity dependence of the mass loss rate in terms of the Fe- and $\alpha$-element adundances, rather than mean abundances? This may become important at high $Z$, where chemical evolution effects play a role.

KUDRITZKI: I agree completely. This effect certainly may become important and I can easily account for this. The calculations presented here were just the starting point to check zero-order effects and to introduce the new algorithm. I also refer to the papers by Puls et al. (2000) and Vink et al. (2001), which dicuss the effects of changing the ratio of $\alpha$-elements to iron group at low metallicity. 